\title{
PU Vul: 15 Years of Studies, Important Findings and Remaining Mysteries
}

\author{
R.E. Gershberg \\ Crimean Astrophysical Observatory, Nauchny, Crimea, Ukraine
}

In autumn 1979 , the young $W$. Wenzel - at this time he was only 50 years old - came to the Byurakan meeting on flare stars and informed the participants about a recently discovered flaring object: the Kuwano-Honda object $=\mathrm{PU}$ Vul. During that time, FU Ori and related stars were the most intriguing variable objects, and PU Vul had shown some similar features such as the rate of brightening and the spectrum at maximum. It turned out to be an extremely interesting object, and the Crimean team has studied it for the last 15 years. Over this time, this team included T.S. Belyakina, N.I. Bondar, K.K. Chuvaev, Yu.S. Efimov, R.E. Gershberg, V.I. Krasnobabtsev, E.P. Pavlenko, P.P. Petrov, I.S. Savanov, N.I. Shakhovskaya, N.M. Shakhovskoy, A.G. Shcherbakov and V.A. Shcherbakov, and we collaborated with V.I. Shenavrin from Russia, with D. Chochol, J. Grygar and L. Hric from former Czechoslovakia and with V.Puirola, I. Tuominen, M. Poutanen and J. Huovelin from Finland.

Now the number of publications on PU Vul approaches one hundred. It was established that $\mathrm{PU}$ Vul is a symbiotic nova, the object among the known variables of this type, whose explosion became observable most recently (with the possible exception of Wakuda's object in Sgr, Grebel et al. 1994), and many details have been observed from the very beginning of the flare. I intend to list the most interesting findings and the most intriguing remaining mysteries of PU Vul.

\section{Important findings}

The binarity of PU Vul was established by two independent observations: (i) in 1979 during brightness maximum a hot stellar spectrum was seen at optical wavelengths, but IR photometry simultaneously showed radiation with a colour temperature of $3200 \mathrm{~K}$ (Bensammar et al. 1980), and (ii) at the descending branch to the 1980-81 brightness minimum (Fig. 1) the spectrum changed from $F$ to $M$ without the appearance of any intermediate spectral classes (Gershberg et al. 1982) and the same $M$ spectrum was found 20 years before the flare (Stephenson 1979). Now it is clear that PU Vul consists of a normal late M giant and a rapidly evolving burst component.

A strong similarity between the spectrum of PU Vul and that of a normal F supergiant was observed in 1979-1983, during the prolonged brightness maxi- 


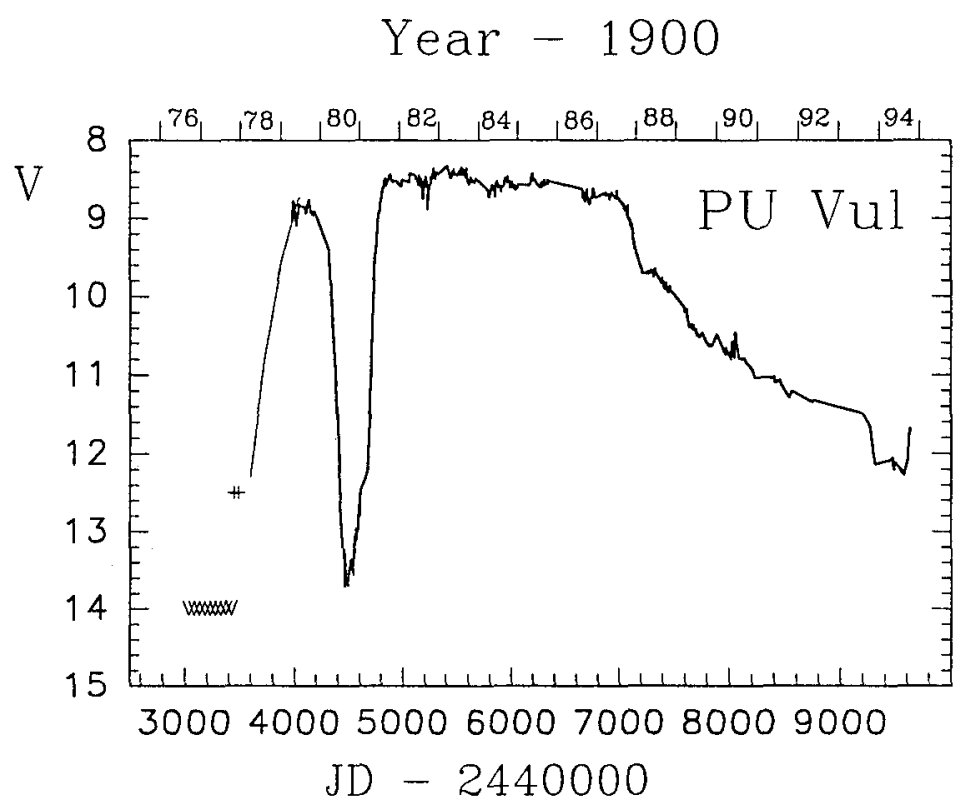

Fig. 1. $V$ lightcurve of PU Vul over the last 15 years.

mum. This similarity was noted from the energy distribution from UV to near IR (Nakagiri \& Yamashita 1982, Friedjung et al. 1984, Belyakina et al. 1985, 1990, Vogel \& Nussbaumer 1992) as well as from relative intensities of absorption lines (Hric et al. 1980, Gershberg et al. 1982, Yamashita et al. 1982, 1983, Kolotilov 1983a, lijima \& Ortolani 1984) and from quantitative analysis by the curve of growth method (Belyakina et al. 1985, Kanamitsu 1991). During these years, PU Vul could not be distinguished from a normal F supergiant by its luminosity, effective temperature, physical parameters and chemical composition of its atmosphere. This fact leads us to consider the existence of a non-homogeneity of high luminosity F stars, and I consider the enigmatic $\epsilon$ Aur a probable candidate for such a mimicry (Gershberg 1990).

A general accordance between observable features of $P U$ Vul and those of theoretical models for the explosion of an accreting white dwarf in a binary system was found, when observations were compared with Iben's (1982) computations (Fig. 2). Iben did not know about the existence of PU Vul when he calculated the models, but Fig. 2 shows the excellent agreement between the observed and the computed luminosity, the effective temperature at brightness maximum, and the rate of brightening before maximum. Theory predicts that the exploded object should describe a loop in the (luminosity - effective temperature) plane near brightness maximum, and in Fig. 3 this loop is presented in the (magnitude - colour index) plane from our data.

The 1980-81 brightness minimum. In the beginning of 1980 , the gradual development of PU Vul along the Iben model track ceased because of a strong brightness decrease. In August-September 1980, PU Vul passed a bright- 




Fig. 2. The theoretical track of an accreting white dwarf (Iben 1982) and the position of PU Vul at brightness maximum in the $(M, \log T)$ plane (Gershberg 1986)

ness minimum with $\Delta V=4 . \cdot 8$, and in September 1981, it had recovered from this decrease. Belyakina et al. (1982b) suggested two possible interpretations of this 500-day minimum: an eclipse of the outbursting component by an $\mathrm{M}$ giant, or the formation of a dust envelope around it, with a subsequent dissipation of the envelope. At that time both these schemes had weak points: Accordingly to Belyakina et al. (1985), the observed duration of the PU Vul brightness decrease and the known size of an $M$ giant lead to an expected orbital period of $200-$ 300 years and a distance between the components of tens of AU. It is unlikely that in such a wide binary a high accretion rate, necessary for a nova-like flare, would be realized. On the other hand, the occurrence of an eclipse of the bursting component in such a system requires that the angle between the line of sight and the orbital plane does not exceed $1^{\circ}$ and that the PU Vul flare took place near conjunction phase. It is obvious that a coincidence of these independent circumstances has an extremely low probability. Recently Vogel \& Nussbaumer (1992) attempted to reanimate the eclipse scheme. However, they have carefully considered the problem from the photometric point of view only and did not take into account the geometrical and dynamical aspects mentioned above, which apparently force to reject this scheme.

The scheme of a non-stable dust envelope was proposed independently by Yamashita et al. (1982) and supported by Friedjung et al. (1984) and Men'shchikov 


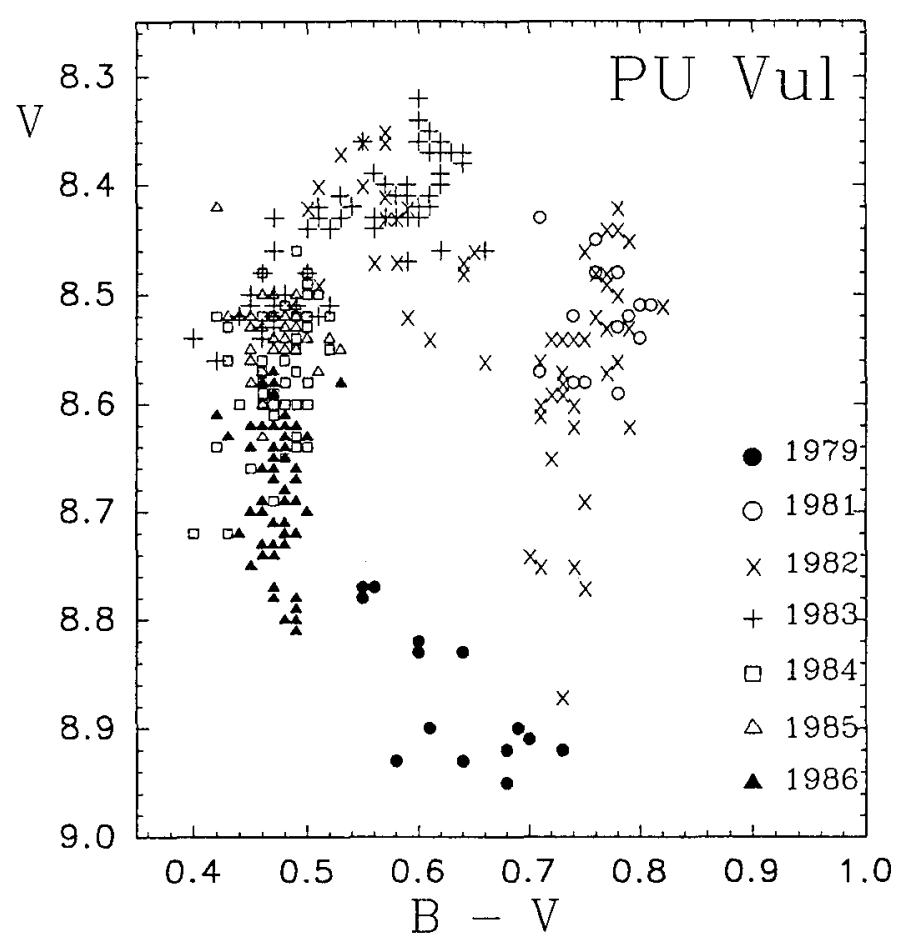

Fig. 3. The observed loop traced by PU Vul in the magnitude - colour index plane near brightness maximum (from Belyakina et al. 1990).

Size in $\mu$

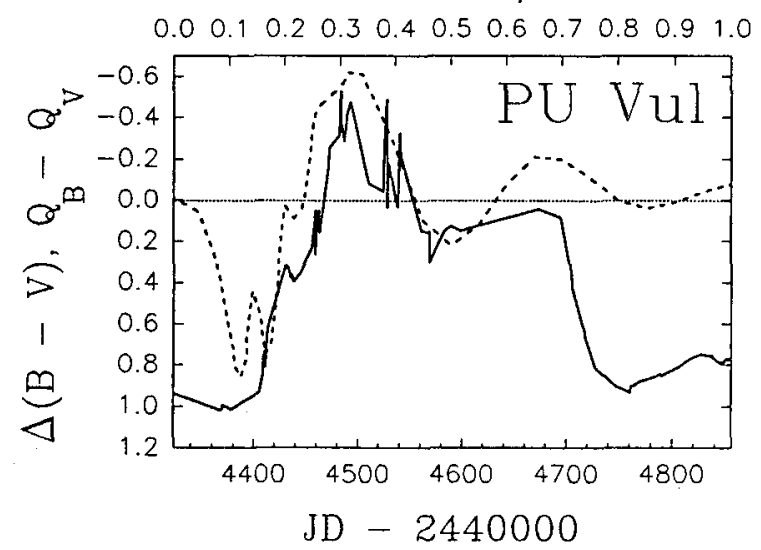

Fig. 4. The observed $\Delta(B-V)$ curve of PU Vul in the deep minimum in 1980-81 (solid line) and the calculated $Q_{B}-Q_{V}$ curve for a spherical silicate dust formation process (dotted line). The lower scale is $\mathrm{JD}$, the upper one is a characteristic size of growing particles (from Efimov 1988a). 
et al. (1985). However, a strong bluening of colour indices at brightness minimum (Belyakina et al. 1982a, Kolotilov \& Belyakina 1982) contradicts the standard idea of the reddening of a star when its brightness decreases due to interstellar dust absorption. This has resulted in critics on the dust scheme (Kenyon 1986, Iijima 1989, Vogel \& Nussbaumer 1992, Sion 1993). Meanwhile, 6 years ago, Efimov (1988a) showed that the observed variations in the colour indices at brightness minimum do not contradict the dust scheme but are a decisive argument in favour of it. To realize this fact, one should consider dust formation as a particle growth process. When conditions for dust formation appear within a circumstellar medium, this process evolves rather quickly, and a given time corresponds to a certain characteristic size of particles. In such a case, brightness minima at different wavelengths should take place at different times, earlier at shorter wavelengths and later at longer ones. Such a sequence was indeed observed. As the result of a monotonous growth of characteristic size of particles, variations of colour indices near brightness minimum should be described by sign-reversing functions. It means that at a certain time a star occulted by a forming dust envelope will be even bluer than before the dust appeared - see Fig. 4. (This concept was successfully applied by Efimov (1988b) to R CrB variables as well, where the cause of non-stable dust envelopes for stellar brightness minima is beyond any doubt.)

Another objection against the dust model of the 500 day brightness minimum of $\mathrm{PU} \mathrm{Vul}$ are the small variations of IR radiation of during this event, while during nova flares accompanied by dust envelope formation, the IR emission usually increases strongly (Kenyon 1986). However, this objection does not account for an essential difference between the considered bursting objects. In novae, a powerful IR excess appears (and is easily detectable) when the donor-star is of low luminosity. In PU Vul, the total IR radiation of the system is mainly determined by the $M$ giant, and the smallness of the IR flux variation does not contradict the dust model (Belyakina et al. 1985). Moreover, these small variations may be even of different signs, depending on their main cause: either due to an intrinsic radiation of dust or to the $M$ giant light reflected from dust - in these cases the total IR radiation of the system would increase, or due to a veiling of the $M$ giant by the dust - in this case the total IR radiation of the system would decrease. As known, the JHK band fluxes from PU Vul decreased by $0.7-0{ }^{m} 4$ (Belyakina et al. 1985), while $\Delta V=4{ }^{\mathrm{m}} 8$.

Thus, objections against the dust model of the brightness minimum in 198081 are rejected and the above model can be accepted. A general scheme of the evolution of PU Vul, including this event, was made by Kanamitsu (1991) and is presented in Fig. 5.

The outflow and the entering to the nebular phase was traced during the slow evolution of PU Vul with unprecendent details. Several stages of this process can be distinguished. The P Cyg profile of $\mathrm{H} \alpha$ - the first evidence of an outflow - was found just after discovery (Mochnacki 1979). Simultaneously detected weakened metallic lines were also considered as due to numerous weak emission lines filling in respective absorptions. These features were observed 

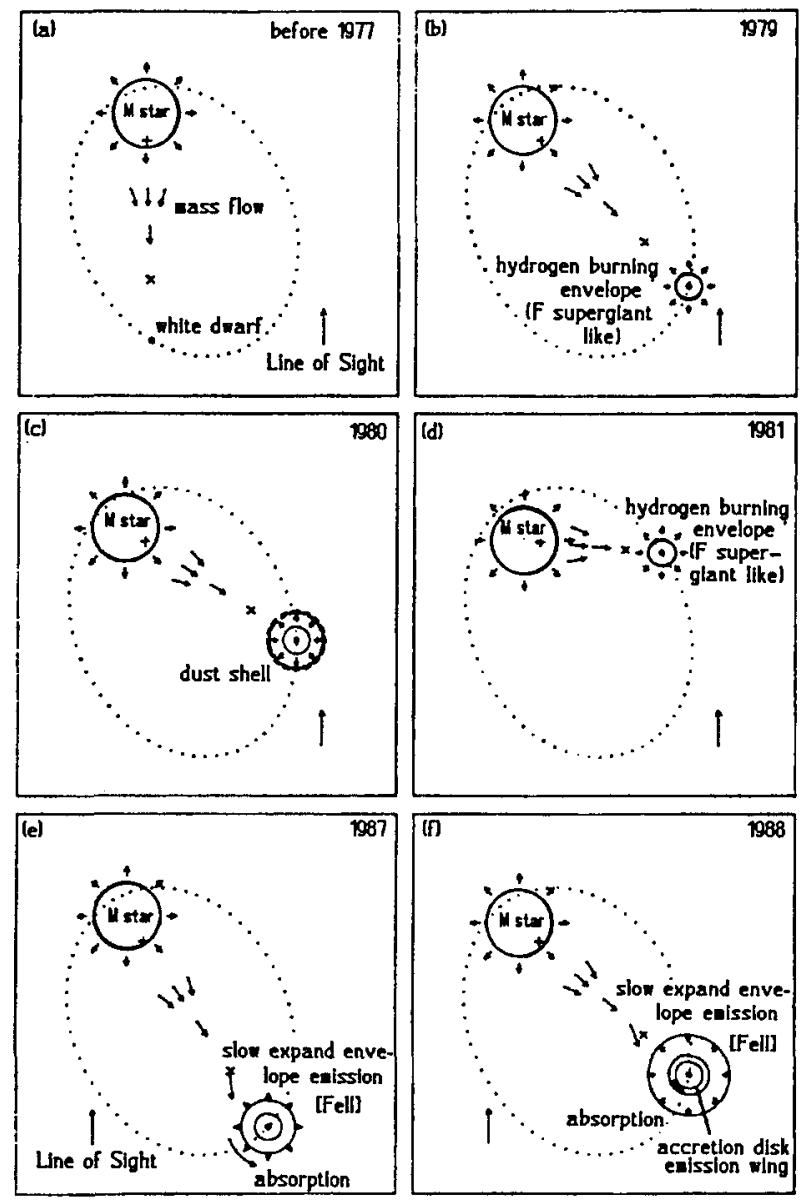

Fig. 5. Schematic plot of the PU Vul binary system in different years (Kanamitsu 1991)

during 6 months (Honda et al. 1979, Yamashita et al. 1982, Kanamitsu et al. 1991). The second episode of the absorption line weakening began in November 1982, two weeks after the brightening of the system by $\Delta U \sim 0.8$ and with the true appearance of emission lines of multiplet series of many elements. In this rather long episode, the line weakening effect was found to be dependent on ionization potentials of respective species. The effect was strongest in July 1984 (Yamashita et al. 1983, 1984, Iijima \& Ortolani 1984, Kolotilov 1984, Kanamitsu et al. 1991). Then, similar weak-line events with hydrogen and Fe II emission lines of different intensities took place in August 1985, in May 1986 and in April 1987 (Kanamitsu 1991, Kanamitsu et al. 1991, Klein et al. 1994).

The last weak-line episode was followed by the appearance of P Cyg line profiles of many species during several months and by the fast flaring of strong emissions of $\mathrm{H}, \mathrm{He}, \mathrm{Fe} I \mathrm{II}$ and [FeII] lines in December 1987. For two years the $\mathrm{H}$ and $\mathrm{Fe}$ emission lines became the main features of the spectrum. This was 
the beginning of the entering in the nebular phase (Iijima 1989, Kanamitsu 1991, Kanamitsu et al. 1991, Klein et al. 1994). During this stage a sequence of appearance of $\mathrm{P} \mathrm{Cyg} \mathrm{line} \mathrm{profiles} \mathrm{of} \mathrm{other} \mathrm{species} \mathrm{and} \mathrm{the} \mathrm{flaring} \mathrm{of} \mathrm{lines}$ of different species depending on their ionization potentials was seen. Then, absorption components of $\mathrm{P}$ Cyg profiles weakened and the spectrum turned into a pure emission spectrum. In November 1989, only the interstellar NaI D lines were seen in absorption.

The nebular phase of PU Vul showed further systematic shifts of emitting species to higher ionization potentials, due to continuing contraction of the burst component in accordance with the increase of effective temperature. During 1988 the $\lambda<1900 \AA$ flux increased by a factor 2 (Vogel \& Nussbaumer 1992), HeI emission became noticeably stronger (Klein et al. 1994) and very wide wings of $\mathrm{H}$ and He lines appeared (Iijima 1989, Sion et al. 1993). From May 1989, the strong UV He II, Si IV, C IV, Si III], CIII] and Al III emission lines became visible (Vogel \& Nussbaumer 1992; Sion et al. 1993; Klein et al. 1994). In the middle of 1990 , emission lines characteristic of WR stars as well as many forbidden lines were registered (Tomov et al. 1991).

\section{Remaining mysteries}

The comparison of the main observational features with theoretical calculations by Iben (1982) for a quasi-static approximation of hydrogen burning on an accreting white dwarf and calculations by Livio et al. (1989) for a hydrodynamical approximation of this process permit to understand the general nature of the PU Vul flare and the evolutionary status of the variable. Accordingly to Duerbeck's (1981) classification, PU Vul corresponds to the Eddington novae, and later it was counted among the symbiotic novae (Viotti 1988). However, these general conclusions leave many open questions for understanding the rich variety of observational data. I intend to list and discuss some of these mysteries.

1. What is the meaning of the small-amplitude quasi-periodical brightness variations in 1979? In that year $\mathrm{PU}$ Vul showed such variations with $\Delta V=00^{\mathrm{m}} 15$ and a quasi-period of about 78 days (Chochol et al. 1981). After the 1980-81 minimum, similar variations of a smaller amplitude and with a shorter time scale were also suggested (Kolotilov \& Belyakina 1982); however, this suggestion was not confirmed (Kolotilov 1983b). On the other hand, nonlinear self-excited oscillations of envelopes of low-massive stars of high luminosity lead to dust formation (Fadeyev 1982, 1983). Is this the case in PU Vul?

2. What is the cause of the small-amplitude brightness variations with unusual colour anomaly $\Delta U<\Delta B \sim \Delta V$, detected in autumn 1982 (Purgathofer $\&$ Schnell 1983; Belyakina et al. 1985)?

3. What is the cause (or causes) of the polarisation of the light of PU Vul? In the long series of polarimetric measurements one can find episodes when the polarization was apparently due to the dust envelope - see, e.g., the data for the 1980-81 minimum. However, when three models - light scattering in a dust envelope, light scattering on free electrons in a magnetized envelope and a spotty photosphere - are used to interpret the complete polarimetric data, none 
of them fits the whole variety of observed wavelength dependence and none of them can be completely rejected. The most intriguing facts are polarimetric data in November 1982 and in September 1983, when during brightenings in the $U$ band the polarization angles essentially changed simultaneously in all the $U B V R I$ bands, while the degrees of polarization were practically constant, and in October-December 1984, when essential variations were detected in $p_{U B V R I}$ without noticeable changes in $\theta_{U B V R I}$ (Belyakina et al. 1985, 1990).

4. Where and why originated the emission spectrum in March-April 1981? At the ascendant branch of the light curve after the deep minimum, the spectrum was quite unique: emission lines of Sc II, LaII, Y II and Nd II were dominant while Fe emission was rather scanty. Only the solar chromosphere (Pierce 1968) and $\eta$ Car and RR Tel (Meinel et al. 1969) show comparable numbers of lines of mentioned ions (Belyakina et al. 1984, 1985). Why was this spectrum so shortlived?

5. Why was the chemical composition of the photosphere so close to that of the normal F supergiant $\gamma$ Cyg in 1979 and 1981? In both cases, there are overabundances of $\mathrm{Y}, \mathrm{Zr}, \mathrm{Ba}$ and La (Belyakina et al. 1985). Does it mean that the accreted hydrogen-rich wind from the $M$ giant did not interact with the upper layers of the hydrogen-depleted accreting white dwarf? What is the cause of chemical abundance variations after 1981? At this time, the PU Vul atmosphere became very similar in this respect to atmospheres of "metallic" stars: excesses of the iron group (excluding Fe itself) and of heavier elements appeared, these excesses being higher with atomic numbers, $\mathrm{Ca}$ and $\mathrm{Sc}$ showed essential deficits (Belyakina et al. 1990, Kanamitsu 1991). Are these variations a real effect? Or are they results of an improper application of the curve of growth method? Or results of a real stratification of chemical abundances along the depth of the quasi-photosphere? Or the consequence of weak-line events?

6. And finally, what are the spatial and kinematic structures of PU Vul? What numbers should be added to Kanamitsu's (1991) scheme to convert this qualitative plot into a quantitative model? To answer this question, we have one of the richest observational data sets and the most scanty conclusive results. Numerous measurements of radial velocity have been carried out (Hric et al. 1980, Yamashita et al. 1982, 1983, 1984, Kolotilov 1983a, 1984, Iijima \& Ortolani 1984, Belyakina et al. 1985, Kanamitsu et al. 1991, Tomov et al. 1991). However, in the very beginning of the measurements an essential heterogeneity was found in the results (Hric et al. 1980). Chochol \& Grygar (1987) have carefully selected lines to calculate an orbit of the binary, but the resulting orbit was not confirmed directly by later observations (Belyakina et al. 1990, Kanamitsu 1991). However, the estimated orbital period of about 8 years turned out to be in agreement with gradual variations of the polarization angle (Belyakina et al. 1990); then, there is observational evidence that in 1981 the bursting component was nearer to us than the $M$ giant (since the dust envelope around the bursting component decreased the IR radiation of the $M$ giant) while in 1989-91 the wind from the $M$ giant produced absorption structures in the spectrum of the bursting component (Sion et al. 1993). 
Many measurements of radial velocity differences between absorption and emission components of P Cyg profiles have been performed (Mochnacki 1979, Yamashita et al. 1982, Vogel \& Nussbaumer 1992, Tomov et al. 1991, Klein et al. 1994). However, these differences were found to be dependent both on time and on the emitting species.

The existing confusing picture of radial velocities is probably partly due to the filling in of absorptions by respective emission lines as well as by the weakline events which are probably of the same nature. For instance, in June 1984 a monotonous change of radial velocities was found along the Balmer series from $0 \mathrm{~km} / \mathrm{s}$ for $\mathrm{H} \gamma$ to $+55 \mathrm{~km} / \mathrm{s}$ for $\mathrm{H} 20$ (Belyakina et al. 1990).

In spite of the uncertainty mentioned above, the observations before the nebular phase were in agreement with theoretical predictions by Livio et al. (1989), that the envelope expansion velocity is about $40 \mathrm{~km} / \mathrm{s}$. At the nebular phase very wide wings of emission line profiles were discovered, and this required new emitting structures: a WR hot wind from the contracting burst component (Tomov et al. 1991) or an accreting disc around this component (Kanamitsu 1991).

In any case, the problem to separate a regular orbital motion and an expansion of the envelope of variable optical thickness is still far from a solution. Several weak-line events in the epoch of the absorption spectrum and very fast - time-scales of several days - profile variations in the nebular phase (Tamura et al. 1992; Efimov et al. 1995) lead to suggest a non-spherical envelope, similar in structure to that of N Cyg 1975 (V1500 Cyg) (Boyarchuk \& Gershberg 1977). However, this makes the search for the structure of PU Vul even more difficult.

Acknowledgement: This research was made possible in part by Grant R2Q000 from the International Science Foundation.

\section{References}

Belyakina T.S., Efimov Yu.S., Pavlenko E.P., Shenavrin V.I., 1982a, Astron. Zh. 59, 1 Belyakina T.S., Gershberg R.E., Efimov Yu.S., et al. 1982b, Astron. Zh. 59, 303

Belyakina T.S., Bondar N.I., Chochol D., Chuvaev K.K., Efimov Yu.S., Gershberg R.E., Grygar J., Hric L., Krasnobabtsev V.I., Petrov P.P., Piirola V., Savanov I.S., Shakhovskaya N.I., Shakhovskoj N.M., Shenavrin V.I., 1984, A\&A 132, 12

Belyakina T.S., Bondar N.I., Gershberg R.E., Efimov Yu.S., Krasnobabtsev V.I., Petrov P.P., Piirola V., Savanov I.S., Chuvaev K.K., Shakhovskaya N.I., Shakhovskoy N.M., Shenavrin V.I., 1985, Bull. Crimean Astrophys. Obs. 72, 3

Belyakina T.S., Bondar N.I., Gershberg R.E., Hric L., Grygar J., Efimov Yu.S., Krasnobabtsev V.I., Piirola V., Poutanen M., Savanov I.S., Tuominen I., Chochol D., Chuvaev K.K., Shakhovskaya N.I., Shakhovskoy N.M., Shenavrin V.I., Shcherbakov A.G., 1990, Bull. Crimean Astrophys. Obs. 81, 28 (A shortened version appeared in A\&A 223 (1989) 119)

Bensammar S., Friedjung M., Assus P., 1980, A\&A 83, 261

Boyarchuk A.A., Gershberg R.E., 1977, Astron. Zh. 54, 488

Chochol D., Hric L., Papousek J., 1981, IBVS 2059

Chochol D., Grygar J., 1987, ApSS 131, 487 
Duerbeck H.W., 1981, PASP 93, 165

Efimov Yu.S., 1988a, Astron. Zh. 65, 807

Efimov Yu.S., 1988b, Astron. Zh. 65, 979

Efimov Yu.S., et al., 1995, this volume, p. 255

Fadeyev Yu.A., 1982, ApSS 86, 143

Fadeyev Yu.A., 1983, ApSS 95, 357

Friedjung M., Ferrari-Toniolo M., Persi P., Altamore A., Cassatella A., Viotti R., 1984, in The future of ultraviolet astronomy based on six years of IUE research, J.M. Mead R.D. Chapman and Y. Kondo (eds.), NASA CP-2349, Washington, p. 305

Gershberg R.E., 1986, Commun. Konkoly Obs. 86, 351

Gershberg R.E., 1990, Astron. Zh. 67, 76

Gershberg R.E., Krasnobabtsev V.I., Petrov P.P., Chuvaev K.K., 1982, Astron. Zh. 59, 6

Grebel E.K., Duerbeck H.W., Greiner J., Richter G.A., 1994, IBVS 4019

Honda M., Ishida K., Noguchi T., Norimoto Y., Nakagiri M., Soyano T., Yamashita Y., 1979, Tokyo Astron. Bull. Second Ser. N 262, 2983

Hric L., Chochol D., Grygar J., 1980, IBVS 1835

Iben I., 1982, ApJ 259, 244

Iijima T., 1989, A\&A 215, 57

Iijima T., Ortolani S., 1984, A\&A 136, 1

Kanamitsu O., 1991, PASJ 43, 225

Kanamitsu O., Yamashita Y., Norimoto Y., Watanabe E., Yutani M., 1991, PASJ 43, 523

Kenyon S.J., 1986, AJ 91, 563

Klein A., Bruch A., Luthardt R., 1994, A\&AS 104, 99

Kolotilov E.A., 1983a, Astron. Zh. 60, 324

Kolotilov E.A., 1983b, Pisma Astron. Zh. 9, 486

Kolotilov E.A., 1984, Pisma Astron. Zh. 10, 284

Kolotilov E.A., Belyakina T.S., 1982, IBVS 2097

Livio M., Prialnik D., Regev O., 1989, ApJ 341, 299

Mochnacki S., 1979, IAU Circ. 3350

Meinel A.B., Aventi A.F., Stockton M.W., 1969, Catalog of emission lines in astrophysical objects, Univ. Arizona, Tucson

Men'shchikov A.B., Tutukov A.V., Shustov B.M., Ergma E.V., 1985, Pisma Astron. Zh. 11, 529

Nakagiri M., Yamashita Y., 1980, Tokyo Astron. Bull. Second Ser. N 263, 2993

Pierce A.K., 1968, ApJS 17, 1

Purgathofer A., Schnell A., 1983, IBVS 2264

Sion E.M., 1993, AJ 106, 298

Sion E.M., Shore S.N., Ready C.J., Scheible M.P., 1993, AJ 106, 2118

Stephenson C.B., 1979, IAU Circ. 3356

Tamura S., Kanamitsu O., Yamashita Y., 1992, PASJ 44, 543

Tomov T., Zamanov R., Miev L., Mikolajewski M., Georgiev L., 1991, MNRAS 252, 31

Viotti R., 1988, in The symbiotic phenomenon, J. Mikolajewska, M. Friedjung, S.J. Kenyon and R. Viotti (eds.), Kluwer, Dordrecht, p. 269

Vogel M., Nussbaumer H., 1992, A\&A 259, 525

Yamashita Y., Maehara H., Norimito Y., 1982, PASJ 34, 269

Yamashita Y., Norimito Y., Yoo K.H., 1983, PASJ 35, 521

Yamashita Y., Norimoto Y., Watanabe E., 1984 (priv. comm.) 